\title{
Radar Based Detection and Tracking of Objects under Poor Visibility Conditions
}

\author{
Alexander Ens ${ }^{1}$, Christian Zech ${ }^{2}$, Axel Hülsmann ${ }^{2}$, Leopold Georgi ${ }^{3}$, Karl-Friedrich Becker ${ }^{3}$ \\ ${ }^{1}$ Fraunhofer IPA (Nobelstr. 12, Stuttgart, Germany), \\ alexander.ens@ipa.fraunhofer.de \\ ${ }^{2}$ Fraunhofer IAF (Tullastr. 72, Freiburg, Germany) \\ ${ }^{3}$ Fraunhofer IZM, (Gustav-Meyer-Allee 25, Berlin, Germany)
}

\begin{abstract}
Places which have to be monitored permanently (such as security sectors, industrial areas or power plants) are facing the problem of large-area monitoring. Optical security cameras can observe such areas under good lighting and visibility conditions, but objects might get invisible when fog, darkness or smoke reduces the visibility.

This problem can be solved with millimeter-wave radars with very high slew rates of the chirp signal. The radar has a detection range of above $100 \mathrm{~m}$ and can determine the distance and speed of objects inside the observation area very precisely. For most applications, the position of objects is more important than their color or size.
\end{abstract}

Key words: large-area monitoring, fast chirp, smoke, fog, W-band radar, RF-PCB

\section{Introduction}

Instead of observing a security area with optical or infrared cameras, the presented radar system uses high frequency millimeter-waves of about $96 \mathrm{GHz}$. A camera (without time-of-light measurement) can only generate 2-dimensional pictures without depth resolution. In contrast, reflected radar signals can encode the distance and speed of objects by measuring frequency changes.

The developed radar module contains an onboard signal generation and onboard antenna, as well as the entire signal processing in the size of a pack of cigarettes. The electronic substrates are made by standard PCB (printed circuit board) technology, allowing easy scalability for industrial mass production. Thanks to the compact dimensions and the waterproof antenna, the radar can be fixed discretely on facades or other outdoor areas. An advantage to optical or infrared light is that millimeter-waves are able to penetrate fog and smoke with very low absorption due to their larger wavelength. Furthermore, the necessary transmitter output power can be kept minimal by using low-noise GaAs-based mHEMT (metamorphic high electron mobility transistor) technology. The integrated signal processing unit is able to detect objects and outputs their distance and speed, so that no further image processing is required.

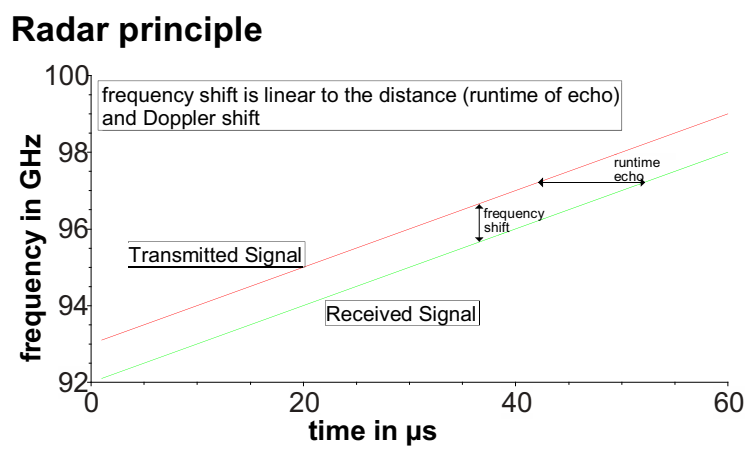

Fig. 1. Relation of the transmitted and received signal of a FMCW radar.

There are two main radar designs. One is the pulse radar, measuring the runtime between the transmission and the reception of a frequency pulse, which is reflected by an object. Thereby, the distance of the object can be determined, as well as its velocity, by analyzing the Doppler shift of the received signal. The second radar design uses a continuous wave, whose frequency is modulated, resulting in a FMCW (frequency modulated continuous wave) radar (fig. 1).

The pulse radar suffers in signal power, caused by the short time pulses to measure near 


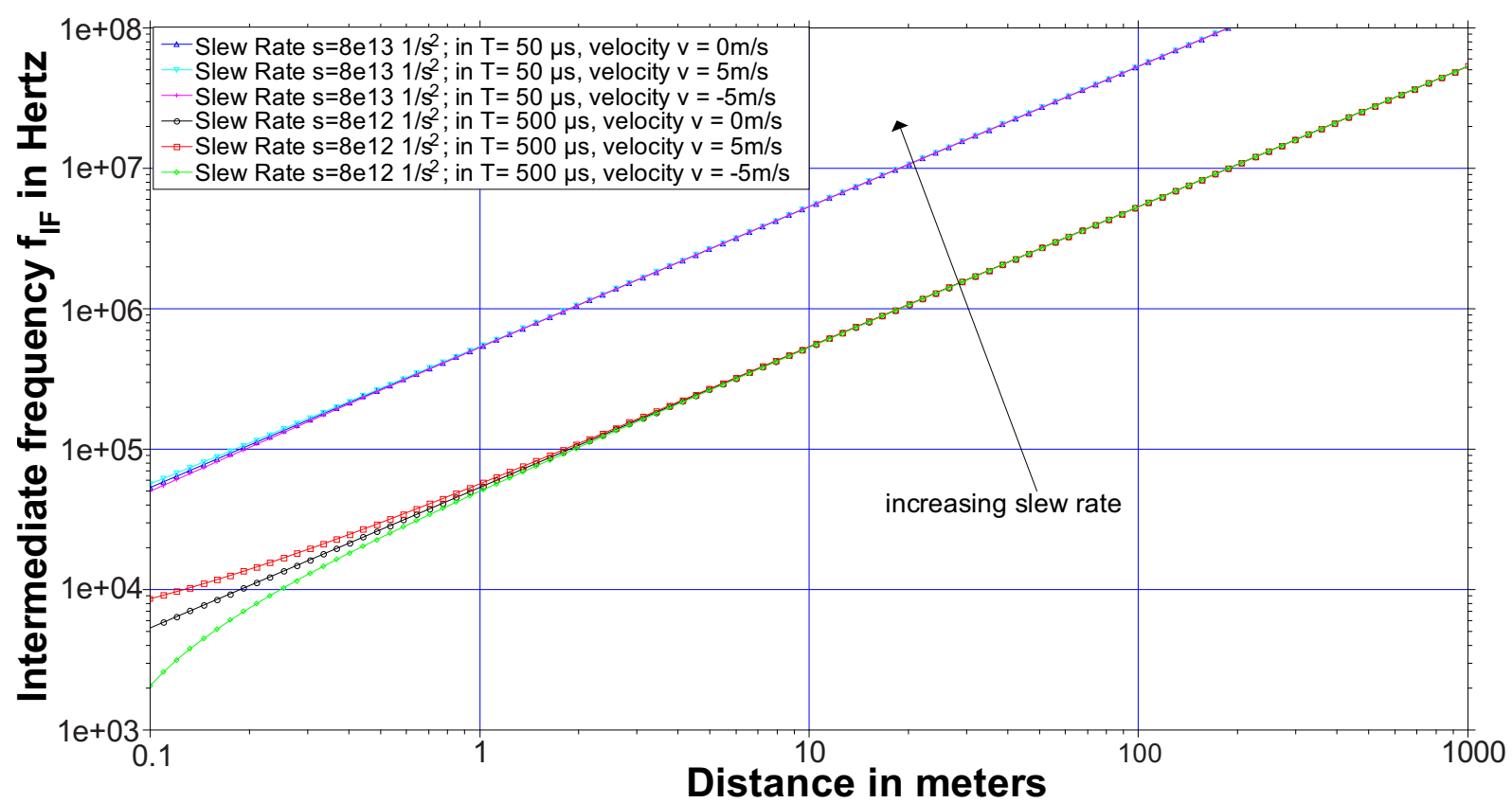

Fig. 2. The received intermediate frequency of an object depends on the distance its distance and velocity as wells as the slew rate of the transmitted FMCW radar signal.

distances. The FMCW principle suffers of clutter, e.g. reflections from steady objects. To overcome these disadvantages, both methods can be combined [1,2]. The slew rate of the chirp sequence has to be very high and therefore the duration of the transmission time is short (fig. 2). To realize this approach, the FMCW radar equation for the received intermediate frequency (IF) has to be analyzed

$$
f_{I F}=\frac{2}{c_{0}}\left(s \cdot d+v \cdot f_{c}\right) \text {, }
$$

whereby $c_{0}$ is the speed of light, $d$ the distance of the object, $v$ its velocity, $s$ the slew rate of the transmitted frequency ramp and $f_{c}$ the ramp's center frequency. To separate the distances and velocities of several objects from the received IF, it is necessary to perform multiple FMCW ramps with different slew rates [3, 4].

The approach used in this paper is that, when the product of the slew rate and distance is much higher than the product of the speed and center frequency

$$
s \cdot d \gg v \cdot f_{c},
$$

the $v \cdot f_{c}$ term can be neglected. As a result, the distance can be calculated directly from the IF. Therefore no matching of different chirp sequences is required to determine the velocity and distance of objects. This simplifies the signal processing because the distance of the objects is unique for each measurement. The velocity has been constrained to $5 \mathrm{~m} / \mathrm{s}$ and the center frequency of the ramp is set to $96 \mathrm{GHz}$, resulting in a velocity frequency product of

$$
v \cdot f_{c}=5 \frac{\mathrm{m}}{\mathrm{s}} \cdot 96 \mathrm{GHz}=4.8 \cdot 10^{11} \frac{\mathrm{m}}{\mathrm{s}^{2}} .
$$

On the other hand, the system should be able to measure distances of up to $300 \mathrm{~m}$ with slew rates of $4 \mathrm{GHz} / 500 \mu \mathrm{s}$ :

$$
s \cdot d=\frac{4 \mathrm{GHz}}{500 \mu \mathrm{s}} \cdot 300 \mathrm{~m}=2.4 \cdot 10^{15} \frac{\mathrm{m}}{\mathrm{s}^{2}} .
$$

It can be seen that the slew rate distance product is orders of magnitude higher than the velocity frequency product, whereby the approach is approved. An inaccuracy in distance calculation is made due to the simplification of the analysis. The distance error $\Delta d$ depends on the slew rate and the velocity

$$
\Delta d=\frac{f_{c} \cdot v}{s}=\frac{96 \mathrm{GHz} \cdot 5 \frac{\mathrm{m}}{\mathrm{s}}}{\frac{4 \mathrm{GHz}}{500 \mu \mathrm{s}}} \approx 0.06 \mathrm{~m} .
$$

The difference in received signal power $(\Delta P)$ decreases with the fourth power of the distance range [3]:

$$
\Delta P=10 \cdot \log _{10}\left(d^{4}\right)=40 \cdot \log _{10}\left(d_{\max }-d_{\min }\right)
$$

For a radar system operating at a range from $1 \mathrm{~m}$ to $100 \mathrm{~m}$, a dynamic range of $\Delta P=80 \mathrm{~dB}$ is necessary to handle the signal power differences. The dynamic range of the ADC (Analog to Digital Converter) is typically limited to $60-70 \mathrm{~dB}$ and can be extended by a variable gain amplifier.

\section{Signal Processing}

Fig. 3 shows a process chart of the signal processing. 
At first, a window function is applied on the received signal and the spectrum is calculated by a FFT (fast-Fourier transformation) algorithm. By using an OSGO-CFAR (orderedstatistic greatest-of constant failure alarm ratio), a threshold value for peak detection is calculated for the spectrum. This threshold is adaptive and depends on the signal intensity of the current measurement, as well as a sensitivity value (alarm ratio). The sensitivity can be adjusted by choosing different guard intervals or changing the number of included measurement points. All spectral components, whose values are higher than the CFAR threshold, are identified as peaks for the object detection.

To improve the accuracy of the peak's estimated frequency, a quadratic fitting is performed for each peak and the maximum turning point of the fitting is used as more precise frequency estimation. The distance of an object is calculated from the estimated frequency using the FMCW equation, while neglecting the velocity term. The next instance is a linear constant velocity Kalman-Filter to track the estimated objects. To allocate the measured distances to the tracked distances a mapping is required. This is solved by NearestNeighbor search. Tracking of objects reduces mismatches by predicting the velocity and the next position of the object.

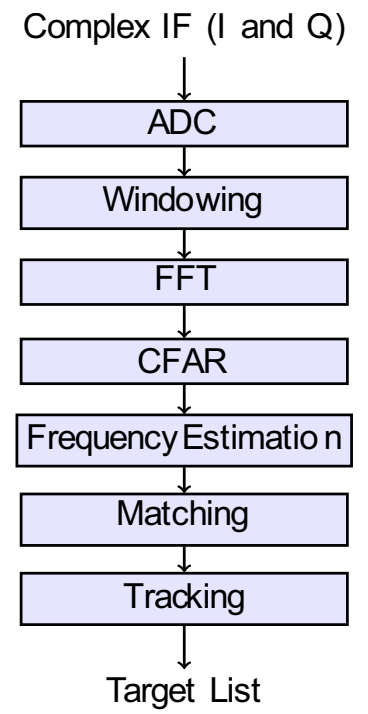

Fig. 2. Signal processing path of FMCW radar.

\section{RF Front End}

The RF (radio frequency) front end consists of three main parts: the signal generation, a radar MMIC (monolithic microwave integrated circuit) and an antenna, which includes a dielectric lens.
The signal generator is based on a fractional-N PLL (phased-locked loop), which is running at an output frequency of $7.8 \mathrm{GHz}$. The reference path of the PLL consists of a $100 \mathrm{MHz}$ ultra-low noise oscillator, which features a phase noise of $-90 \mathrm{dBc} / \mathrm{Hz}$ at $10 \mathrm{~Hz}$ frequency offset, while the noise floor is as low as $-168 \mathrm{dBc} / \mathrm{Hz}$. Furthermore the PLL offers an integrated sweep generator, which is used to realize the FMCW frequency chirp. The chirp bandwidth is $150 \mathrm{MHz}$ at $7.8 \mathrm{GHz}$, resulting in a total bandwidth of $1.8 \mathrm{GHz}$ after multiplication to $94 \mathrm{GHz}$. The PLL uses a discrete $3^{\text {rd }}$ order passive $100 \mathrm{kHz}$ loop filter to drive an external VCO (voltage-controlled oscillator). The VCO signal is multiplied by two, using a commercial active frequency multiplier in a $3 \times 3 \mathrm{~mm}^{2}$ package, which features an output power of $17 \mathrm{dBm}$. The signal is then attenuated, using a passive VVA (voltage variable attenuator) to drive the input port of the radar MMIC. The total area of the signal generator is less than $2 \times 2 \mathrm{~mm}^{2}$.

The MMIC is based on the $100 \mathrm{~nm}$ mHEMT technology developed at IAF. The single chip circuit is realized on a $50 \mu \mathrm{m}$ thinned GaAs substrate. The chip size is $2 \times 4 \mathrm{~mm}^{2}$. Details of the $100 \mathrm{~nm}$ gate length $\mathrm{mHEMT}$ technology are published at [5]. The schematic of the chip is shown in Fig. 4.

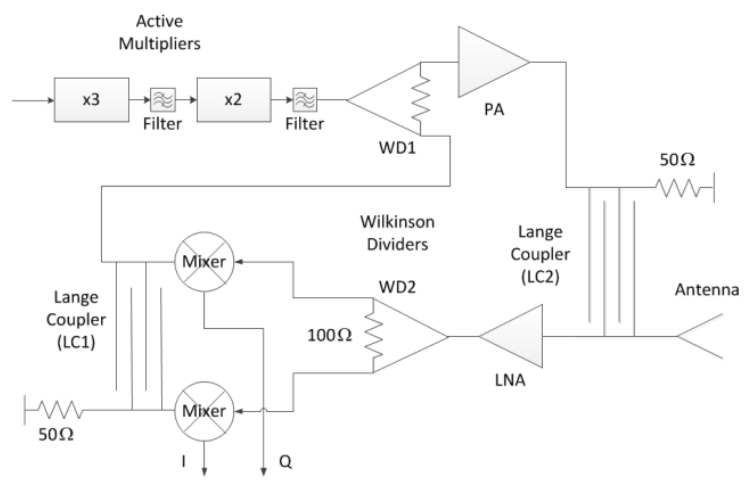

Fig. 4. Schematic of the FMCW MMIC.

The input signal is multiplied by a factor of 6 using two integrated active frequency multipliers $x 3$ and $x 2$. The chirp signal is than split by a Wilkinson divider (WD1) and one part is amplified by a power amplifier (PA). The other part is divided by a Lange coupler (LC1) to support two mixers, which are $90^{\circ}$ out of phase. A second Lange (LC2) coupler separates the transmit $(T x)$ and receive $(R x)$ signal at the antenna port. The insulation between transmit and receive signal is $-20 \mathrm{~dB}$, which is a sufficient compromise, if we take into account that Lange couplers can be integrated on MMICs. The Rx signal is amplified by a LNA and split by a second Wilkinson divider (WD2), to feed the I/Q mixer. The I and $Q$ output 
signals of the mixer are used for later signal processing. A photograph of the chip is shown in Fig. 5.

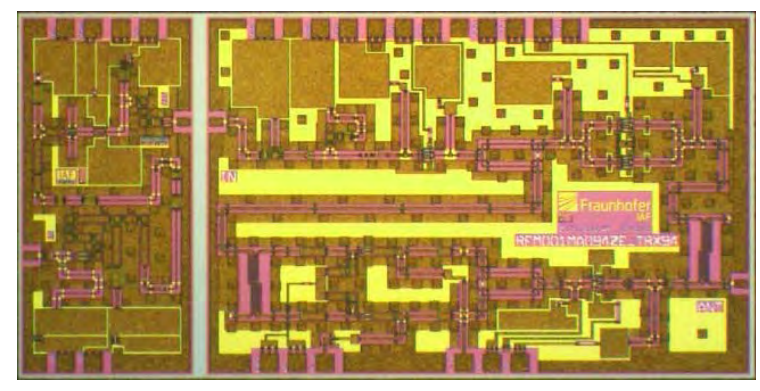

Fig. 5. Photograph of the chip of the GaAs radar MMIC.

The transmit path of the MMIC was characterized by on-wafer measurements. Fig. 6 shows the output power measurements for different input power levels. The MMIC features an output power of up to $8 \mathrm{dBm}$ with an excellent broadband performance from 75 to $105 \mathrm{GHz}$. The limited RF output bandwidth of the radar system is due to the limited bandwidth of the $7.8 \mathrm{GHz} \mathrm{VCO}$.

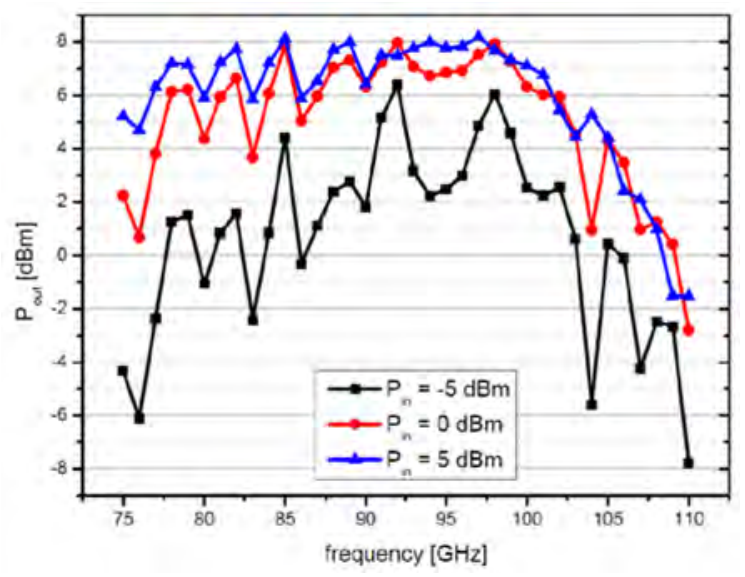

Fig. 6. MMIC output power for different input power level over output frequency.

The antenna port of the MMIC is connected to a GCPW (grounded coplanar waveguide) transmission line on a high-frequency suitable $50 \mu \mathrm{m}$ thin LCP (liquid crystal polymer) substrate (such as Rogers ULTRALAM ${ }^{\circledR} 3850$ or Taconic $\operatorname{TacLam}^{\circledR}$ ), using wire-bond techniques. The geometry of the transmission line has been optimized to a ground-to-ground spacing of $525 \mu \mathrm{m}$, while the center conductor width is $125 \mu \mathrm{m}$. The simulated attenuation of this line is $0.15 \mathrm{~dB} / \mathrm{mm}$. The GCPW line terminates in an antipodal Vivaldi-antenna. The Vivaldi-antenna has been chosen due to the planar feasibility on the two-layer LCP substrate. The top copper layer is used to shape the signal plane of the antenna; the ground plane is realized in the bottom layer. The copper surfaces are gold finished to avoid surface oxidation and to ensure long-time radiation performance.

The center of the antenna is put into the tip of a dielectric cone, which is expanding in the direction of the radiation. To realize application specific aperture angles of the radar system, the front of the cone is shaped as a lens. The dielectric cone, as well as the lens can be produced cost-efficiently as one part, using injection molding technology. High-frequency suitable materials, such as HDPE (high-density polyethylene) or PTFE (polytetrafluoroethylene) can be used. A patent on this kind of antenna design is pending Fehler! Textmarke nicht definiert. A photograph of the Vivaldi-antenna (including the GCPW feeding line) with the dielectric cone and lens is shown in Fig. 7.

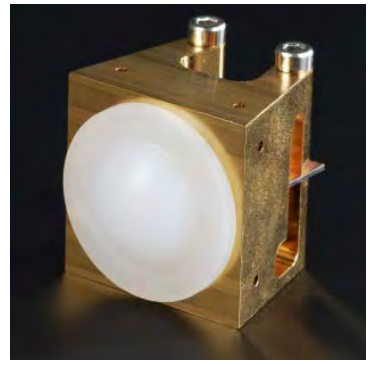

(a)

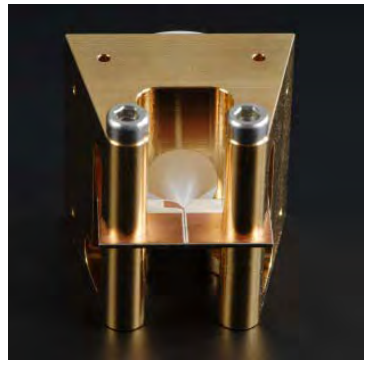

(b)
Fig. 7. Photograph of the front (a) and back (b) of the antenna with dielectric cone and lens.

\section{Technical implementation}

The technical implementation consists of three major parts: The housing with integrated dielectric lens as well as the electronic parts, which are made of two modular PCB-boards. First board contains the digital signal processing unit. Second board is composed of a high-frequency suited Liquid Crystal Polymer (LCP) substrate for the RF-signal paths, laminated onto a FR4 circuit board, forming a 3D-structured hybrid package. The Vivaldiantenna is realized as an edge emitter on a free standing LCP-membrane and the MMIC is integrated into a cavity on the LCP. This combination of standard low-cost materials and processes with minimized commitment to special RF-materials makes the device applicable for cost-effective mass production. Fig. 8 shows the described setup schematically.

The LCP was chosen amongst other commercially available RF-suited substrates, due to the good RF-properties (dielectric constant: 2.9 at $10 \mathrm{GHz}$, dissipation factor: 0.0025 at $10 \mathrm{GHz}$ ) paired with mechanical properties which give the opportunity to fabricate substrates as thin as $50 \mu \mathrm{m}$ which is important for avoiding substrate modes. 


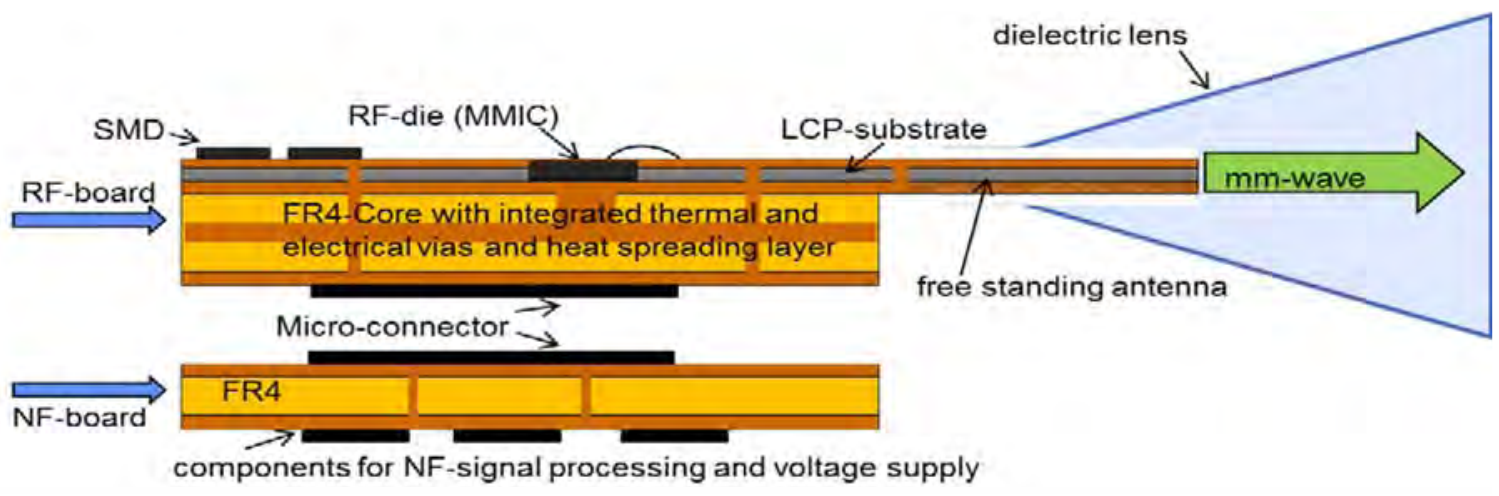

Fig. 8. Schematically overview of the radar-board.

\section{Process}

Standard PCB-processes need to be optimized regarding good RF-performance, several issues have to be respected [7]:

Surface quality has to be superior for RF-leads and antenna as well as wire bonding and dieattach. Thus the copper etching process was optimized for perpendicular walls and very smooth surface (Fig. 9). Afterwards a surface finish suitable for wire-bonding was deposited with a LCP-adapted ENEPIG (Electroless Nickel / Electroless Palladium / Immersion Gold) process.

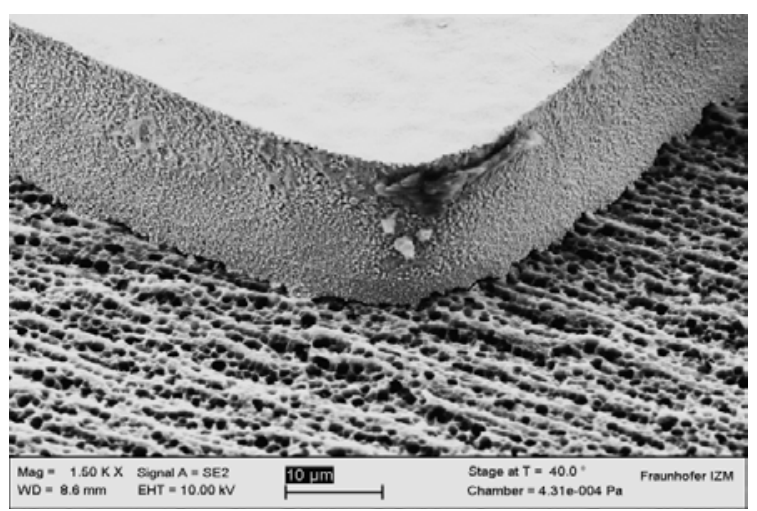

Fig. 9. Electron microscopy of the edge of a Vivaldi-antenna on LCP-Substrate.

The hybrid assembly of LCP and FR4 in combination with the free standing LCPmembrane needs very controlled execution of PCB-cavity-milling, prepreg lamination and electroplating. Furthermore, alternating operation of laser- and milling-processes is required to compensate for the different mechanical properties of LCP and FR4.

For minimal loss of the $94 \mathrm{GHz}$ signal, the wirebonds have to be as short as possible. For this reason, the MMIC is placed and adhesive bonded very accurately into a laser structured cavity. Aim is to minimize the gap and the difference in the level between the border of the chip and the edge of the cavity. By the use of standard dispense and die-bond equipment, it was possible to achieve typical gap width $10 \mu \mathrm{m}$ and level variations of $10 \mu \mathrm{m}$ (Fig. 10). With this geometry, automated wire bonding with a length of $150 \mu \mathrm{m}$ was possible.

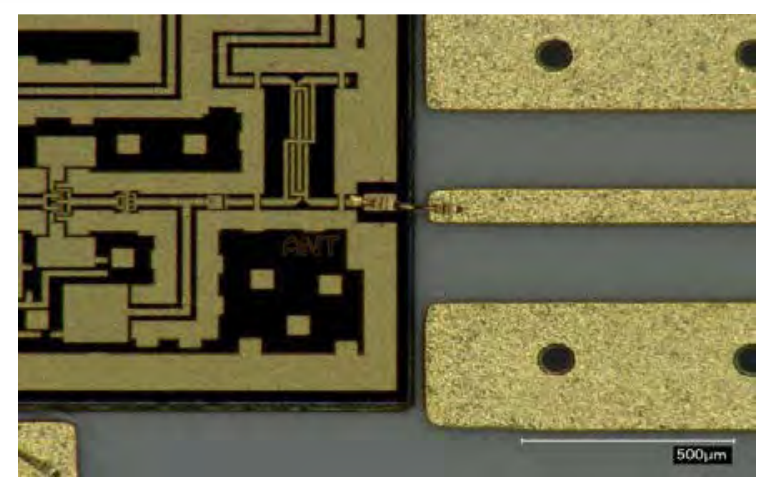

Fig. 10. Assembled MMIC in laser cavity. Wirebond length is approx. $150 \mu \mathrm{m}$.

Thermal management plays an important role in system design. Especially the components for high frequency generation, like frequency converters, tend to have quite high thermal dissipation losses. The heat generated is conducted away via direct contact between the components and the housing on the one hand and on the other hand through vias connecting the component with a heat spreading layer, laminated into the middle of the substrate stack for mechanical stability.

[1] A. Ludloff: „Praxiswissen Radar und Radarsingalverarbeitung“, Vieweg, 3rd Edition 2002, ISBN: 3-528-26568-X

[2] N. Levanon, E. Mozeson: "Radar signals", John Wiley 1st Edition 2004, ISBN: 0-471-47378-2

[3] H. Winner, S. Hakuli, G. Wolf: „Handbuch Fahrerassistenzsysteme“", Vieweg+Teubner, 2nd Edition 2012, ISBN: 978-8348-457-9

[4] A. W. Rihaczek: "Principles of high-resolution radar", MrGraw-Hill, 1st Edition 1969, ISBN: 0754-32106-9

[5] A. Tessmann, I. Kallfass, A. Leuther, H. Massler, M. Schlechtweg, and O. Ambacher:

"Metamorphic MMICs for operation beyond 200 $\mathrm{GHz}$ " in European Microwave Integrated Circuit Conference (EUMIC), 2008, pp. 210-213

[6] Radarantenne, Deutsches Patent- und Markenamt, DE 102012220531.4

[7] K.-F. Becker, M. Koch, R. Kahle, T. Braun, L. Böttcher, A. Ostmann, J. Kostelnik, F. Ebling, E. Noack, J.P. Sommer, M. Richter, M. Schneider, H. Reichl: „Embedding Technology Development for a $77 \mathrm{GHz}$ Automotive Radar System" in MST Kongress 2009, Berlin 